Takahashi, A. Mechanism Of Deuteron Cluster Fusion By EQPET Model. in Tenth International Conference on Cold Fusion. 2003. Cambridge, MA: LENR-CANR.org. This paper was presented at the 10th International Conference on Cold Fusion. It may be different from the version published by World Scientific, Inc (2003) in the official Proceedings of the conference.

\title{
Mechanism Of Deuteron Cluster Fusion By EQPET Model
}

\author{
Akito Takahashi \\ Osaka University, Yamadaoka 2-1, Osaka, 565-0871 Japan, \\ akito@nucl.eng.osaka-u.ac.jp
}

\begin{abstract}
The present paper makes deepened modeling for the mechanism of transient Bose-type condensation for $4 \mathrm{D}$ and $8 \mathrm{D}$ clusters with electrons from Pd 4d-shell in PdDx lattice dynamics. Formation of quadruplet $\mathrm{e}^{*}(4,4)$ electronic quasi-particle and octal-coupling $\mathrm{e}^{*}(8,8)$ around lattice focal points with D-clusters which realize super screening for $d$ - $d$ and $d$-cluster Coulomb barrier is modeled for tetrahedral and octahedral symmetric condensations and discussed. Based on the electronic quasiparticle expansion theory (EQPET), modal fusion rates which are composed of 2D, 3D, 4D and 8D fusion rates with characteristic values for each mode are estimated for the tetrahedral and octahedral symmetric condensations. Based on given modal fusion rates we can estimate macroscopic cluster fusion rates $(\mathrm{f} / \mathrm{s} / \mathrm{cc})$ by knowing time-averaged values of D-cluster densities. Several to few hundreds w/cc level fusion rates for 4D and 8D can be foreseen with major ash of ${ }^{4} \mathrm{He}$ and a variety of secondary transmutation reactions, and neutron production rate by $2 \mathrm{D}$ fusion will be with $10-12$ orders smaller magnitude than that of ${ }^{4} \mathrm{He}$ production. Neutrons are therefore hardly visible in deuteron cluster fusion in condensed matter.
\end{abstract}

\section{Introduction}

The model of tetrahedral (TRF) and octahedral (ORF) resonance fusion under transient Bose condensation (TBC) of deuteron cluster at PdDx lattice focal points was proposed ${ }^{1}$ at ICCF9. To make quantitative estimation for modeled reaction process, the electronic quasi-particle expansion theory (EQPET) was proposed ${ }^{2}$ to formulate mathematical equations and some numerical results were given. Calculated results, based on the single particle approximation for $\mathrm{e}^{*}\left(\mathrm{~m} * / \mathrm{m}_{\mathrm{e}}, \mathrm{Z}\right)$ electronic quasi-particle states, revealed that dde* $(2,2)$ transient molecular state had fusion rate of $10^{-20} \mathrm{f} / \mathrm{s} / 2$ Dpair and $10^{-17} \mathrm{f} / \mathrm{s} / 4 \mathrm{D}$-cluster. Strong enhancement of $8 \mathrm{D}$ cluster fusion was also speculated by octahedral condensation. It was concluded that major reactions were 4D cluster fusion to produce two $23.8 \mathrm{MeV}{ }^{4} \mathrm{He}$-particles and $8 \mathrm{D}$ cluster fusion to produce two $47.6 \mathrm{MeV}{ }^{8} \mathrm{Be}-$ particles. These high energy charged particles would make secondary transmutation reactions ${ }^{3}$, including fission, with metal elements and heavier sample elements. Local full $(x=1)$ or over-loading condition $(x>1)$ of PdDx lattice and phonon-excitation of $\mathrm{D}$ in lattice were supposed to require for stimulation of TRF and ORF reactions.

Microscopic mechanism of TBC for tetrahedral symmetric condensation (TSC) is proposed and discussed in the present paper. TBC/TSC generates transient electronic quasi-particles(TEQP), e* $(2,2)$ Cooper pair, especially $\mathrm{e}^{*}(4,4)$ quadruplet (and moreover $\mathrm{e}^{*}(8,8)$ octal-coupling for ORF) in dynamically "frozen state" condensation at room temperature, which enables super-screening for Coulomb interaction of d-d pair and dcluster. Therefore, TBC/TSC is a kind of dynamic Bose-type condensation and different from the atom condensation at absolute "zero" degree K (Bose-Einstein condensation).

Quantum-mechanical treatment of TRF (tetrahedral resonance fusion) by TBC/TSC is formulated by the EQPET modeling to implement quantitative studies on reaction rates.

\section{Tetrahedral Symmetric Condensation}

Under the stimulation of D-phonon excitation in PdDx (suppose locally $\mathrm{x}=1$ ) lattice, transient 4D-cluster will be formed with certain probability ${ }^{4}$. We know the trapping periodical potential height for D in PdDx lattice is about $0.22 \mathrm{eV}$, and we use kinetic energy of deuteron $\mathrm{Ed}=0.22 \mathrm{eV}$ for numerical estimation in the following. To keep charge neutral sate in average, orthogonal combination of two transient $\mathrm{D}_{2}$ molecules will be formed when 4 deuterons are squeezing from O-sites to central T-sites taking (conveying) 4 electrons from $\mathrm{Pd} 4 \mathrm{~d}$-shell 
(conduction band), as shown in Fig.1, under the TSC condition and the cluster of 4D+'s and 4e-'s makes a regular cube as shown in Fig.2.

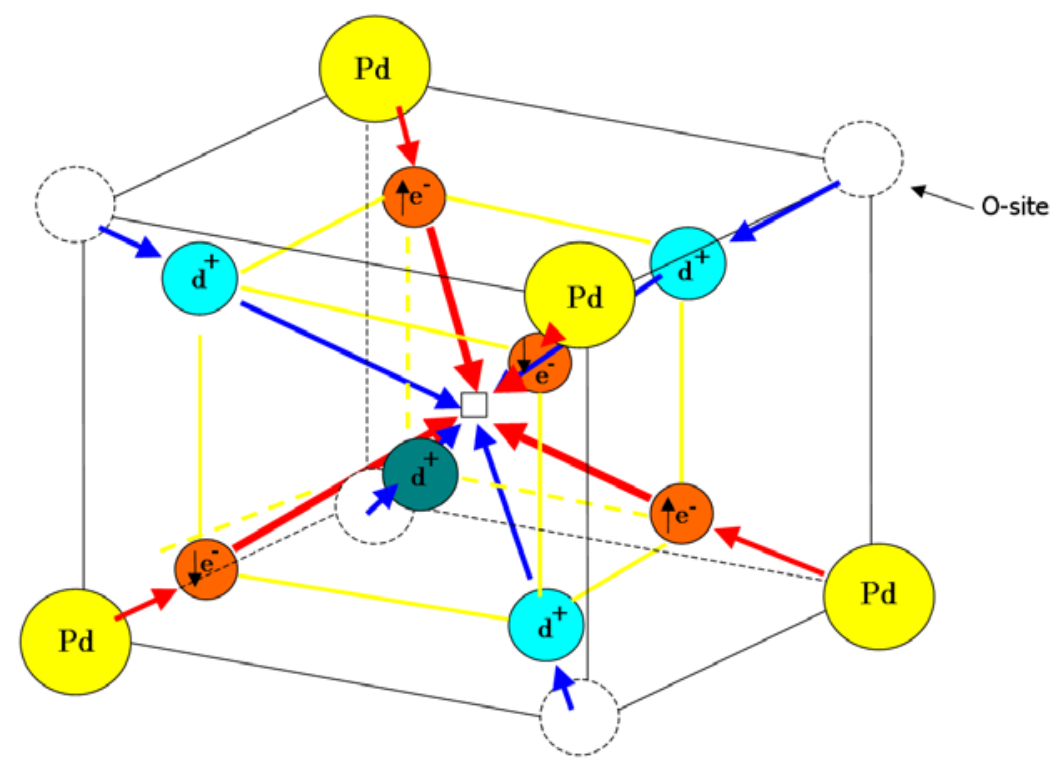

Figure 1. Tetrahedral condensation of deuterons in PdD lattice

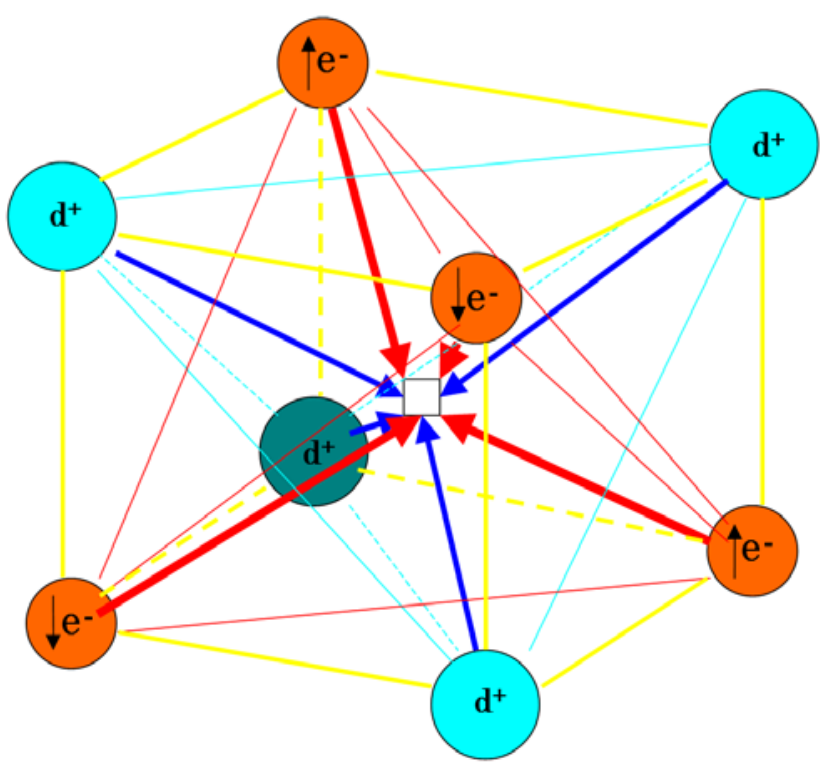

Figure 2. Classical view of Tetrahedral Symmetric Condensation (TSC)

In Figure 2, the arrow on electron indicates the direction of spin. Transient orthogonal combination of two $\mathrm{D}_{2}$ (dede) molecules (upper and lower), 4 deuterons and 4 electrons of which take positions at face centers of regular octahedron, make a regular cube. The 4 deuterons makes a regular tetrahedron, and the 4 electrons does a regular tetrahedron too. Supposing same velocities for all deuterons and their conveying electrons, averaged charge-neutral balance is kept for $4 \mathrm{~d}+$ 's and $4 \mathrm{e}$-'s and freedom of motion is "frozen" except for the direction from O-sites to the central T-site. In the view of classical mechanics, 4 deuterons and 4 electrons squeeze ultimately and condense into a point at the T-site. However, due to the fermion nature of electron, superposition of pairing electron wave functions must be satisfied to avoid Pauli's exclusion rule. When the combination of electron pairs with opposite spins happens, for 4 out of 6 e- pairs, as drawn in Figure 2, we can consider that 
TBC (transient Bose-condensation)/TSC(tetrahedral symmetric condensation) is attained. In this moment, 4 electrons making a regular tetrahedron with orthogonal two Cooper-like pairs (upper and lower) e*(2,2)s converges to a quadruplet $\mathrm{e}^{*}(4,4)$ which can be approximated as an single particle state for calculating trapping (screening) potential for 4D cluster.

We can extend the idea to the octahedral symmetric condensation for local $\mathrm{PdD}_{2}$ lattice to meet the TBC/OSC(octahedral symmetric condensation ${ }^{1}$ ) condition and generation of octal coupling of electrons $\mathrm{e}^{*}(8,8)$ with 4 up-spin electrons in upper side and 4 down spin electrons in lower side with each 4 deuterons.

In figure 2, the sum momentum vector of upper two deuterons exists on a same line of the sum momentum vector (opposite in mirror symmetric direction) of lower two deuterons. The situation is the same for electrons in Figure 3. Therefore the three-dimensional TSC squeezing motion can be transformed to a two-dimensional motion as illustrated in Figure 2 of reference ${ }^{3}$. Statistical weight (combination probability) of Cooper-like pair with opposite momentum and spin electrons can be counted in the two dimensional map as 12/16, and weight for $\mathrm{e}^{*}(4,4)$ as $2 / 16$. In the D-phonon excitation from O-site to T-site, TSC condition is not always satisfied, but with sufficient phonon excitation $\left(\mathrm{Ed}=0.22 \mathrm{eV}\right.$ for instance) we have combination probability $1 / 4^{12}$. Consequently for TBC/TSC, formation probabilities are $18.75 \%$ for $\mathrm{e}^{*}(2,2), 3.12 \%$ for $\mathrm{e}^{*}(4,4)$, and $88.13 \%$ for normal electron state. These weights will be used later in EQPET calculation. In figures 3 and 4, 3-dimensional features of $\mathrm{e}^{*}(4,4)$ and $\mathrm{e}^{*}(8,8)$ are drawn, respectively.

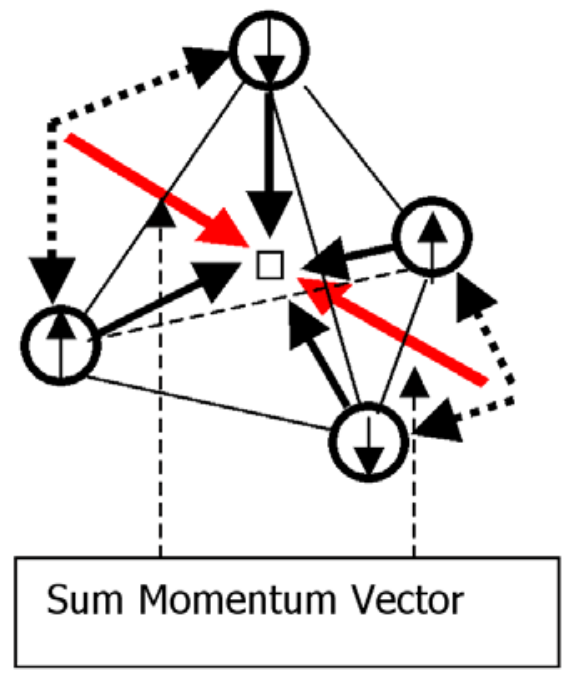

Figure 3. Quadruplet e* $(4,4)$

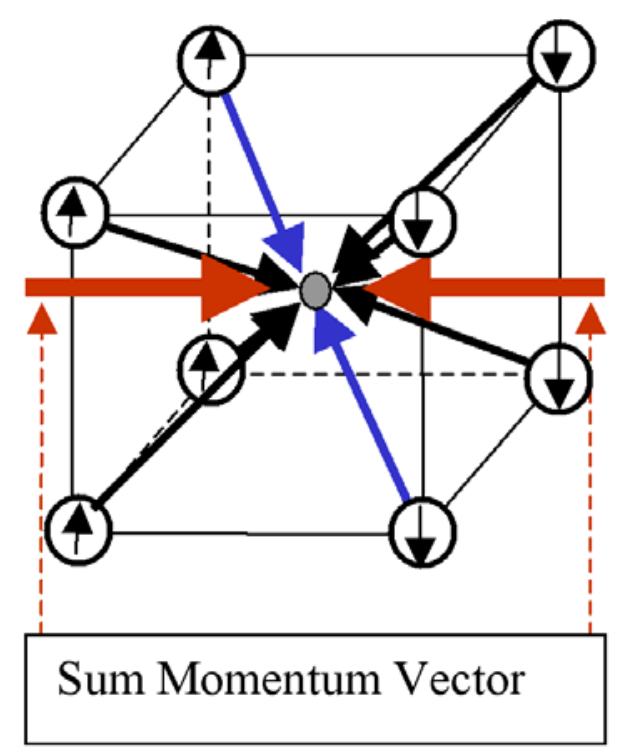

Figure 4. Octal coupling $\mathrm{e}^{*}(8,8)$

In TBC/TSC, we expect that transient D-cluster state can be analyzed as superposition of transient d-d molecular states, e.g., dde and ddee with normal electron $(1,1)$ states and dde* and dde* ${ }^{*}$ with transient electronic quasi-particles (TEQP), $(2,2),(4,4)$ and $(8,8)$ states. Hence we apply an expansion theory of total Dcluster wave function with the linear combination of solved wave functions for transient molecular states ${ }^{2}$.

$$
\left|\Psi_{\mathrm{N}}>=\mathrm{a}_{1}\right| \Psi_{(1,1) \mathrm{G}}>+\mathrm{a}_{2}\left|\Psi_{(2,2) \mathrm{G}}>+\mathrm{a}_{4}\right| \Psi_{(4,4) \mathrm{G}}>+\mathrm{a}_{8} \mid \Psi_{(8,8) \mathrm{G}}>
$$

Screening potentials Vs(R) for each EQPET molecule dde* and dde*e* were formulated and calculated ${ }^{2}$ using the variational method as extension of solutions for dde $\left(\mathrm{D}_{2}^{+}\right.$molecule $)$and ddee $\left(\mathrm{D}_{2}\right.$ molecule) given in text books of quantum mechanics ${ }^{5}$ as

$$
\mathrm{V}_{\mathrm{s}\left(\mathrm{m}^{*} / \mathrm{me}, \mathrm{e}^{* / e}\right)}(\mathrm{R})=\mathrm{V}_{\mathrm{h}}+\mathrm{e}^{2} / \mathrm{R}+(\mathrm{J}+\mathrm{K}) /(1+\Delta)
$$

Where the Coulomb integral $\mathrm{J}$, the exchange integral $\mathrm{K}$ and the non-orthogonal integral $\Delta$ are given in our previous paper ${ }^{2}$.

We also solved an atomic de* system to obtain ground state energy $\mathrm{V}_{\mathrm{h}}$ as:

$\mathrm{V}_{\mathrm{h}}=-13.6 \mathrm{Z}^{2} /\left(\mathrm{m}_{\mathrm{e}} / \mathrm{m}^{*}\right)$ 
For dde* $\mathrm{e}^{*}$ molecule state with double electrons or $\mathrm{e}^{*}$ 's, we also extend the solution for ddee given in the text ${ }^{5}$ and we have obtained screened potential function $\mathrm{V}_{\mathrm{se}^{*} \mathrm{e}^{*}}$ as:

$\mathrm{V}_{\mathrm{se}^{*} \mathrm{e}^{*}}(\mathrm{R})=2 \mathrm{~V}_{\mathrm{h}}+\mathrm{e}^{2} / \mathrm{R}+\left(2 \mathrm{~J}+\mathrm{J}^{\prime}+2 \Delta \mathrm{K}+\mathrm{K}^{\prime}\right) /\left(1+\Delta^{2}\right)$

Here the cross-Coulomb integral J' and cross exchange integral $\mathrm{K}^{\prime}$ are given elsewhere ${ }^{2}$.

For fusion rates of multi-body $(\mathrm{nD})$ reactions in a $\mathrm{D}$-cluster was defined ${ }^{1}$ as:

$$
\lambda_{\mathrm{nd}}=\left[\mathrm{vS} \mathrm{nd}_{\mathrm{d}}\left(\mathrm{E}_{\mathrm{d}}\right) / \mathrm{E}_{\mathrm{d}}\right] \exp \left(-\mathrm{n} \Gamma_{\mathrm{n}}\left(\mathrm{E}_{\mathrm{d}}\right)\right)
$$

Here we have assumed that a simultaneous multi-body fusion process can be approximately treated by the very fast cascade process of two body barrier penetration, namely we treat as $(D+(D+(D+D)))$ to be $4 D$ process. So that we need to first calculate a Gamow integral $\Gamma_{n}(E d)$ for a d-d pair that is given in our previous paper ${ }^{2}$ for $n D$ cluster.

$\mathrm{S}(0)$ values which are intrinsic nuclear fusion cross sections by strong interaction can have multi-body "selective resonance" and hence very large cross sections for 4D (TRF) and 8D (ORF) due to 3-diensional strong enhancement of contact surface area for charged-pion exchange which equivalently enhance fusion cross sections ${ }^{1}$.

\section{Super Screening by TEQP}

Barrier factors, $\exp \left(-\mathrm{n} \Gamma_{\mathrm{n}}\left(\mathrm{E}_{\mathrm{d}}\right)\right)$, and microscopic fusion rates $\lambda_{\mathrm{nd}}$ were given previously ${ }^{2}$, but referred in Table 1 due to some additional notions.

In calculating Gamow integral for barrier penetration, b-value becomes key parameter to scale screening effect, i.e., enhancement of barrier factor., as shown in figure 5.

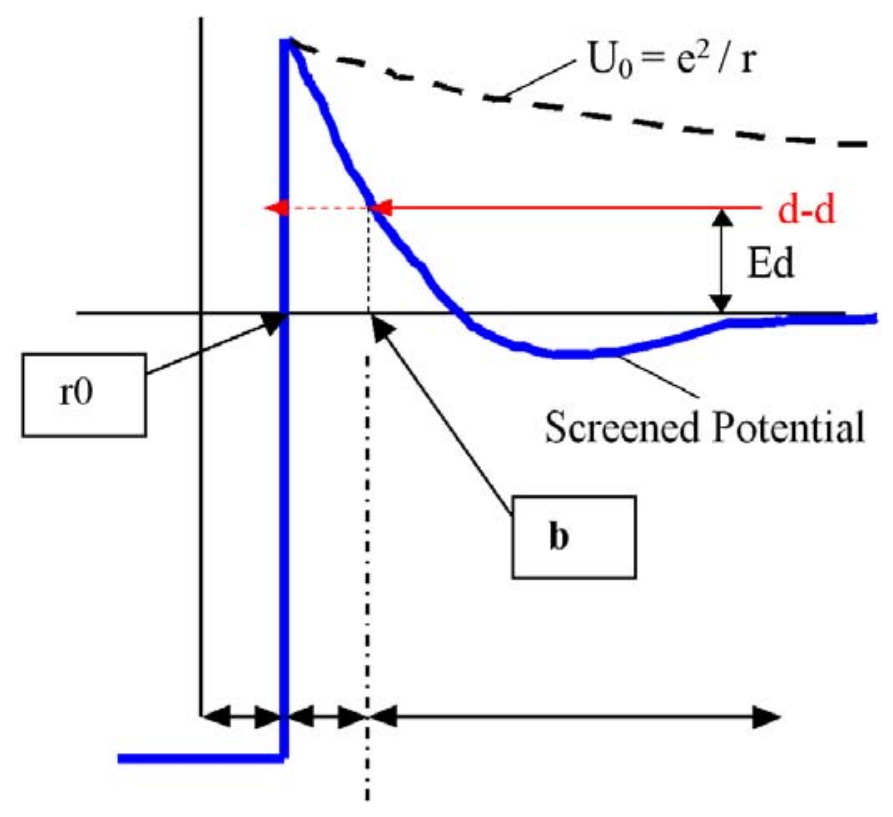

Figure 5. Three steps to calculate D-cluster fusion rates, cluster formation with screened potential, barrier penetration and inter-nuclear fusion 
Table 1 Calculated barrier factors (BF) and microscopic Fusion Rates (FR)

\section{Barrier Factors (BF) and Fusion Rates (FR)}

\begin{tabular}{|c|c|c|c|c|c|c|c|c|}
\hline & & & & $E d=$ & $2 \mathrm{eV}$ & & & \\
\hline \multirow[t]{2}{*}{$\left(\mathrm{m} *, \mathrm{e}^{*}\right)$} & \multicolumn{4}{|c|}{ Barrier Factor } & \multicolumn{4}{|c|}{ Fusion Rate (f/s/cl) } \\
\hline & 2D & 3D & 4D & 8D & 2D & 3D & 4D & 8D \\
\hline$(\mathbf{0 , 0 )}$ & E-1685 & & & & E-1697 & & & \\
\hline$(1,1)$ & E-125 & E-187 & E-250 & E-500 & E-137 & E-193 & E-252 & E-499 \\
\hline$(2,1)$ & E-53 & E-80 & E-106 & E-212 & E-65 & E-86 & E-108 & E-211 \\
\hline$(2,2)$ & E-7 & E-11 & E-15 & E-30 & E-20 & E-17 & E-17 & E-29 \\
\hline$(4,4)$ & $(3 \mathrm{E}-4)$ & E-5 & E-7 & E-14 & $(\mathrm{E}-16)$ & E-11 & E-9 & E-13 \\
\hline$(8,8)$ & $(4 E-1)$ & $(2 \mathrm{E}-1)$ & (1E-1) & 2E-2 & (E-13) & (E-7) & (E-3) & E-1 \\
\hline
\end{tabular}

In Table $1,\left(\mathrm{~m}^{*}, \mathrm{e}^{*}\right)$ means $\left(\mathrm{m}^{*} / \mathrm{m}_{\mathrm{e}}, \mathrm{Z}\right)$ value for TEQP. As shown in Table-1, screening effect by a normal electron $(1,1)$ for dde transient molecule is very great as $\mathrm{BF}=1 \mathrm{E}-125$, compared with the bare reaction $(0,0)$ with $\mathrm{BF}=1 \mathrm{E}-1685$. However, fusion rate $1 \mathrm{E}-137$ is too small to detect by multiplying deuteron density order $1 \mathrm{E} 22$ $\mathrm{d} /$ cc. When $\mathrm{e}^{*}(2,2)$ is formed, BF enhances very much to be in the order of $1 \mathrm{E}-7$ and microscopic $2 \mathrm{D}(\mathrm{d}-\mathrm{d})$ fusion rate increases to be $1 \mathrm{E}-20$ which would give macroscopic rate of $100 \mathrm{f} / \mathrm{s} / \mathrm{cc}$ assuming order of $1 \mathrm{E} 22 \mathrm{~d}-\mathrm{d}$ pairs/cc. This level of neutron emission well corresponds to claimed neutron emission rates in $\mathrm{D}_{2} \mathrm{O} / \mathrm{Pd}$ or TiDx CF experiments ${ }^{6,7}$.

Interestingly, with generation of $\mathrm{e}^{*}(2,2), 3 \mathrm{D}$ and $4 \mathrm{D}$ microscopic fusion rates of the order of $1 \mathrm{E}-17$ have exceeded the $2 \mathrm{D}$ fusion rate as previously discussed ${ }^{2}$. The generation of $\mathrm{e}^{*}(4,4)$ quadruplet of TEQP can give really super screening effect on barrier factors: $1 \mathrm{E}-7$ for $4 \mathrm{D}$ process and very large microscopic fusion rate as 1E-9. However, the barrier factor $3 \mathrm{E}-4$ for dde* $(4,4)$ is "virtual", since only dddde* $(4,4)$ state holds for $\mathrm{TBC} / \mathrm{TSC}$ condition as shown in Figure 2. Similarly, virtual BF values and FR values are shown with parentheses in Table 1.

Qualitative understanding of "super-screening" can be obtained by looking screening potentials with bparameters for fusion by transient motion (a kind of collision process). Screened potentials for $\mathrm{e}^{*}(2,2)$ and $\mathrm{e}^{*}(4,4)$ are shown in Figures 6 and 7, respectively.

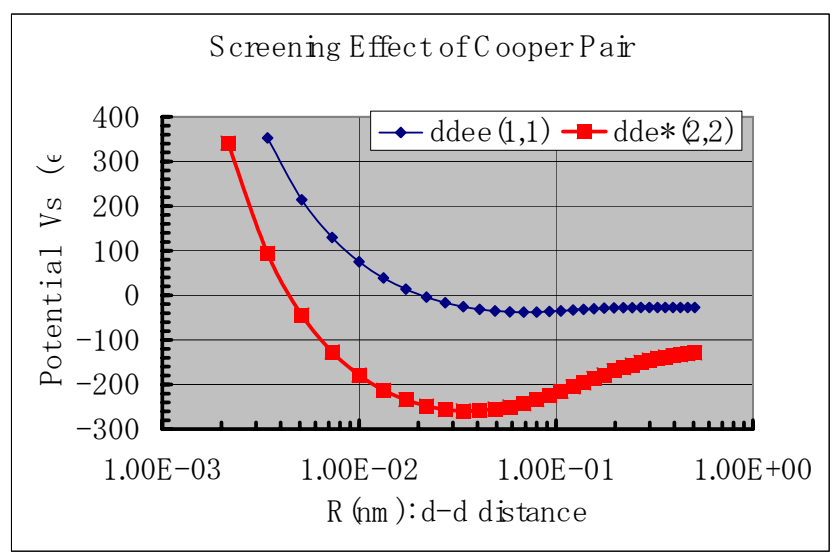

Figure 6. Screened potential for $\mathrm{D}_{2}$ molecule with $\mathrm{b}(1,1)=20 \mathrm{pm}$, compared with dde $*(2,2)$ molecule with $\mathrm{b}(2,2)=4 \mathrm{pm}$ 
For dynamic barrier penetration problem, assuming relative deuteron kinetic energy $\mathrm{Ed}=0.22 \mathrm{eV}$, Gamow integral is done from the b-value where Vs crosses "zero" potential value to the r0 value of about $5 \mathrm{fm}$ for contact surface of nuclear fusion (range of pion). For $\mathrm{D}_{2}$ molecule, b-value is $20 \mathrm{pm}$. For the dde*(2,2) EQPET molecule, b-value is $4 \mathrm{pm}$ which drastically decrease Gamow integral and hence increase BF value. This effect is very large as comparable to mass $=10 \mathrm{~m}_{\mathrm{e}}$ heavy fermion ${ }^{2}$.

When quadruplet $\mathrm{e}^{*}(4,4)$ is formed in TBC/TSC, virtual screening potential for dde* $(4,4)$ molecule becomes very deep as $-2.4 \mathrm{keV}$, as shown in Figure 7 , and b-value becomes $0.45 \mathrm{pm}(450 \mathrm{fm})$. When $\mathrm{e}^{*}(8,8)$ is formed by $\mathrm{TBC} / \mathrm{OSC}$, b-value is very small as $60 \mathrm{fm}$ and approaching to the range of strong interaction. Therefore, the generation of quadruplet and octal coupling of TEQP in TRF and ORF process is essential for super screening. The screening effect by $\mathrm{e}^{*}(4,4)$ is comparable to that of muon (mass $\left.207 \mathrm{~m}_{\mathrm{e}}\right)^{2}$.

Table 1 gives fusion rates for EQPET molecules. In actual condition of TBC/TSC, we have to consider statistical weights by quantum mechanics. Because of this reason, we define modal fusion rates.

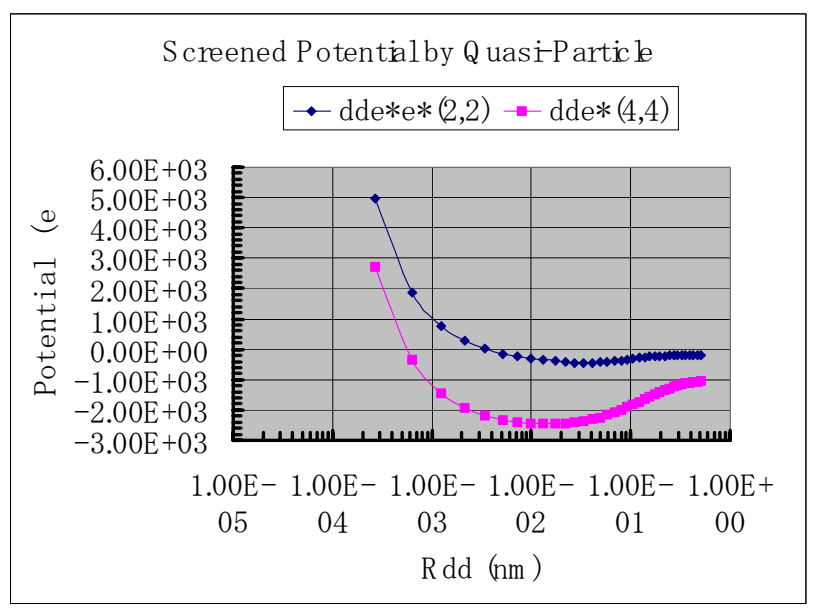

Figure 7. Screening potential for dde*(4,4) molecule with b-value of $0.45 \mathrm{pm}$, compared with dde*e*(2,2) potential with $b$-value of $3 \mathrm{pm}$.

\section{Modal Fusion Rates}

Since 4D cluster of TSC contains partial clusters of 2D, 3D and 4D with transient electronic quasi-particles (TEQP), TBC/TSC condensation produces 2D, 3D and 4D nuclear fusion reaction rates with certain weights as specific values. Using EQPET wave function Eq.(1) and fusion rates for $(1,1),(2,2),(4,4)$ and $(8,8)$ EQPET molecules in Table 1, modal fusion rates are defined as:

$\lambda_{2 \mathrm{~d}}=\mathrm{a}_{1}^{2} \lambda_{2 \mathrm{~d}}(1,1)+\mathrm{a}_{2}^{2} \lambda_{2 \mathrm{~d}}(2,2)$

$\lambda_{3 \mathrm{~d}}=\mathrm{a}_{1}^{2} \lambda_{3 \mathrm{~d}}(1,1)+\mathrm{a}_{2}^{2} \lambda_{3 \mathrm{~d}}(2,2)+\mathrm{c}_{4} \mathrm{a}_{4}^{2} \lambda_{3 \mathrm{~d}}(4,4)$

$\lambda_{4 \mathrm{~d}}=\mathrm{a}_{1}^{2} \lambda_{4 \mathrm{~d}}(1,1)+\mathrm{a}_{2}^{2} \lambda_{4 \mathrm{~d}}(2,2)+\mathrm{a}_{4}^{2} \lambda_{4 \mathrm{~d}}(4,4)$

$\lambda_{8 \mathrm{~d}}=\mathrm{a}_{1}^{2} \lambda_{8 \mathrm{~d}}(1,1)+\mathrm{a}_{2}^{2} \lambda_{8 \mathrm{~d}}(2,2)+\mathrm{a}_{4}^{2} \lambda_{8 \mathrm{~d}}(4,4)+\mathrm{a}_{8}{ }^{2} \lambda_{8 \mathrm{~d}}(8,8)$

Using statistically estimated weights, namely combination probability in the previous section, we calculated modal fusion rates for tetrahedral condensation TBC/TSC. Similarly we have obtained modal fusion rates for octahedral condensation TBC/OSC. Results are shown in Table 2. 
Table 2. Modal fusion rates for tetrahedral condensation (TSC) and octahedral condensation (OSC)

\begin{tabular}{|c|c|c|}
\hline Item & $\begin{array}{l}\text { Tetrahedral Condensation } \\
\text { (TBC/TSC) }\end{array}$ & $\begin{array}{l}\text { Octahedral Condensation } \\
\text { (TBC/OSC) }\end{array}$ \\
\hline $\mathrm{a}_{1}{ }^{2}$ & 0.781 & 0.843 \\
\hline $\mathrm{a}_{2}{ }^{2}$ & 0.187 & 0.079 \\
\hline $\mathrm{a}_{4}{ }^{2}$ & 0.0312 & 0.070 \\
\hline $\mathrm{a}_{8}{ }^{2}$ & 0.0000 & 0.0078 \\
\hline$\lambda_{2 \mathrm{~d}}$ & $1.9 \mathrm{E}-21 \quad(\mathrm{f} / \mathrm{s} / \mathrm{cl})$ & $7.9 \mathrm{E}-22$ \\
\hline$\lambda_{3 \mathrm{~d}}$ & $1.6 \mathrm{E}-13 \quad(\mathrm{f} / \mathrm{s} / \mathrm{cl})$ & $3.5 \mathrm{E}-13$ \\
\hline$\lambda_{4 \mathrm{~d}}$ & $3.1 \mathrm{E}-11 \quad(\mathrm{f} / \mathrm{s} / \mathrm{cl})$ & $7.0 \mathrm{E}-11$ \\
\hline$\lambda_{8 \mathrm{~d}}$ & & $7.8 \mathrm{E}-4$ \\
\hline
\end{tabular}

Using Table 2, we can calculate actual fusion rates for certain D-phonon excited condition, by estimating $\mathrm{nD}$ cluster formation probability ${ }^{4}$ (the first step in Figure 5) in atomic motion level in PdDx.

With $\mathrm{Ed}=0.22 \mathrm{eV}$, all lattice deuterons are excited to going out of periodical trapping potential in lattice, and we may assume cluster density of the order of $1 \mathrm{E} 22 \mathrm{cl} / \mathrm{cc}$. With this condition and modal fusion rates in table 2 for TSC, we obtain 4D fusion rate as 3E11 f/c/cc. 4D fusion produces two $23.8 \mathrm{MeV}$ (highest energy, and possible scheme for lower energy emission was discussed in ref-4) ${ }^{4} \mathrm{He}$ particles which slow down in PdDx with soft X-ray (less than $20 \mathrm{keV}$ ) mostly by convey electrons ${ }^{2}$, and deposit heat as 1 watt per 1E11 4D fusion reactions. Triton and ${ }^{3} \mathrm{He}$ are produced by $3 \mathrm{D}$ fusion with $1.6 \mathrm{E} 8 \mathrm{t}$ or ${ }^{3} \mathrm{He} / \mathrm{s} / \mathrm{cc}$. Neutrons are produced by $2 \mathrm{D}$ fusion with $10 \mathrm{n} / \mathrm{s} / \mathrm{cc}$ which is hardly detectable level with conventional neutron detector systems. Consequently, major claims of $\mathrm{CF}$ experiments, namely ${ }^{4} \mathrm{He}$ production with heat, without visible neutron emission can be explained by TBC/TSC with EQPET analysis.

Fusion rate for $8 \mathrm{D}$ in TBC/OSC is very high as $7.8 \mathrm{E}-4$. If we have $1 \mathrm{E} 16 \mathrm{cl} / \mathrm{cc}$ (namely the order of one ppm) density of local overloading condition $(\mathrm{PdDx}, \mathrm{x}=2)$, we can have 78 watts/cc heat level with high energy ${ }^{8} \mathrm{Be}$ production. High energy ${ }^{8} \mathrm{Be}$ has short life as $1.6 \mathrm{E}-17 \mathrm{~s}$, but will make secondary capture (fusion) reaction ${ }^{3}$ with host (or sample) heavier elements during their ranges before breaking up to two alpha-particles $\left({ }^{4} \mathrm{He}\right)$. Thus, mass- 8 and charge- 4 increased transmutation (two- $\alpha$ adding transmutation) claimed by Imamura $^{8}$ can be explained with the present theory.

High energy (23.8 MeV at highest) alpha-particles by 4D fusion can overcome Coulomb barrier (about 10 $\mathrm{MeV}$ or so) of interaction with host (Pd) elements and makes mass- 4 and charge- 2 increased transmutation reactions. Production of $\mathrm{Cd}$ isotopes as claimed by Karabut ${ }^{9}$ and Mizuno ${ }^{10}$ can be explained with this scenario.

\section{Concluding Remarks}

Transient Bose-type condensation (TBC) of D-cluster under tetrahedral symmetric (TSC) or octahedral symmetric (OSC) condensation has been proposed as microscopic mechanism of super screening of d-d nuclear interaction with transient electronic quasi-particle (TEQP) generation. The EQPET (electronic quasi-particle expansion theory) method is a useful tool for making numerical analysis.

Modal fusion rates for TSC and OSC conditions were defined and numerically estimated. By knowing timeaveraged D-cluster density in metal-deuteride system under stimulation of higher phonon excited states for D in lattice, we can estimate macroscopic fusion rates in $\mathrm{f} / \mathrm{s} / \mathrm{cc}$ unit.

Further study on excitation curve of TSC probability as a function of D-phonon energy in MDx system is expected.

Major products of D-cluster fusion by TSC and OSC are ${ }^{4} \mathrm{He}$ which can reach power level of 100 watts/cc, and neutron emission yields will be $10-12$ or more orders lower than ${ }^{4} \mathrm{He}$ production rate. Transmutation reactions with selected mass and charge transfer can also be explained by the present model. Fission product analysis by alpha-induced reaction for Pd are shown in this conference by our colleagues ${ }^{11}$. 


\section{References}

1 A. Takahashi: Proc. ICCF9, Beijing 2002, 343 (2002)

2 A. Takahashi : Proc. JCF4, Iwate Japan 2002, 74 (2002)

3 A. Takahashi, Y. Iwamura, S. Kuribayashi: Proc. JCF4, Iwate Japan 2002, 79 (2002)

4 A. Takahashi, et al.: Fusion Technology, 27, 71 (1995)

$5 \mathrm{~J}$. Pauking and A. Wilson: Introduction to quantum mechanics with application to chemistry, McGraw-Hill (1935)

6 A. Takahashi, et al: J. Nuclear Science and Technology, 27, 7, 663 (1990)

7 S. Jones, et al.: Nature, 338, 373 (1989)

8 Y. Iwamura, et al: Jpn. J. Appl. Phys., 41, 4642 (2002)

9 A. Karabut, et al: Proc. ICCF9, Beijing China 2002, 151 (2002)

10 T. Mizuno et al: Denkikagaku, 64, 1160 (1996)

11 M. Ohata, A. Takahashi: this conference

12 M. Ohta, A. Takahashi: Proc. ICCF8, Italian Physical Society, Conf. Proc. Vol.70, .403 (2000) 